\title{
Cytoreductive Surgery and Hyperthermic Intraperitoneal Chemotherapy in Gastrointestinal Stromal Tumors Induced Sarcomatosis
}

\author{
Areej N Shihabi*, Ali Al Dameh, Sobhy Mohammed Amer, Huma Darr and Sadir Alrawi \\ Department of Surgical Oncology center, Al Zahra Cancer Center, United Arab Emirates
}

Submission: August 07, 2017; Published: August 21, 2017

*Corresponding author: Areej N Shihabi, Department of Surgical Oncology center, Al Zahra Cancer Center, United Arab Emirates, Tel: 00971-56-509-4660; Email: areej-shihabi@hotmail.com

\begin{abstract}
The aim of this article review is to summarize our current knowledge and advances in the management of gastrointestinal stromal tumors (GISTs).The primary aim of this article is to determine the surgical outcomes of Cytoreductive surgery and Hyperthermic intraperitoneal chemotherapy (CRS/HIPEC) procedures on patients with GIST induced sarcomatosis, the effects on progression free survival and overall survivaland to define the impact of tyrosine kinase inhibitors, TKI resistance on overall survival of patients treated with CRS/HIPEC.
\end{abstract}

Keywords: Gastrointestinal stromal tumors GIST; Cytoreductive surgery; Hyperthermic intraperitoneal chemotherapy (CRS/HIPEC); Peritoneal sarcomatosis; Tyrosine kinase inhibitors TKI

Abbreviations: GISTs: Gastrointestinal Stromal Tumors; GIT: Gastrointestinal Tract; CRS/HIPEC: Cytoreductive Surgery Hyperthermic Intraperitoneal Chemotherapy; ATP: Adenosine Triphosphate; PFS: Progression Free Survival; HIPEC: Hyperthermic intraperitoneal chemotherapy; IP: Intraperitoneal; RPLP: Retroperitoneal Liposarcoma

\section{Introduction}

Gastrointestinal stromal tumors (GISTs) are uncommon mesenchymal tumors that arise mainly in the gastrointestinal tract (GIT). Gastrointestinal stromal tumor (GIST) has the highest incidence and prevalence of gastrointestinal tract sarcomas, accounting for approximately five per cent of all mesenchymal tumors. After the discovery of gain-of-function mutations in 1998, the c-KIT proto-oncogene made these tumors distinguished from other subtypes of mesenchymal tumor. KIT is a $145-\mathrm{kDa}$ glycoprotein. The KIT receptor has an epitope on the extra-cellular domain, CD117, which can be detected by immunohistochemical staining. Lymph node metastases are uncommon in GIST. Distant metastases most commonly of the peritoneum, omentum, mesentery and the liver. GISTs have a high tendency to seed and hence intraperitoneal or even scar metastases are known to occur [1-4, 14].

\section{Treatment of GIST}

I. Surgical: Surgery with complete surgical resection remains the mainstay of GIST treatment and the treatment of choice in localized or potentially respectable disease. In 2002, tyrosine kinase inhibitors (TKIs) has completely changed the management way in GIST [1-3,11,14].

II. Tyrosine kinase inhibitors (TKIs): Imatinib mesylate is a tyrosine kinase inhibitor antagonist to ABL, BCR-ABL, KIT, PDGFRA, PDGFRB and CSF1R. Its structure mimics adenosine triphosphate (ATP) and it works by binding competitively to the ATP binding site of the target kinases. Which will prevent substrate phosphorylation and signaling, resulting in inhibiting proliferation and survival. Patients with advanced GIST started on imatinib have shown a 35\%-49\% 9 year survival. The presence and the type of KIT or PDGFRA mutation status are predictive of response to imatinib. Although surgery is the mainstay of treatment, it does not cure GIST alone.

In $85 \%$ of cases, complete resection is possible but $50 \%$ of patients will develop recurrence or metastasisfollowing complete resection. The 5-year survival rate is approximately50\%, with a median time of 2 years for recurrence after resection of primary high-risk GIST. Adjuvant imatinib has been shown to improve PFS and OS in surgically treated patients. (PFS: progression free survival, OS overall survival) $[1-3,11]$. Sunitinib is a second-line 
agent which is shown to be effective in non-responders cases to imatinib therapy [1-3].

\section{Metastatic (Sarcomatosis) or recurrent disease}

III. Cytoreductive surgery and Hyperthermic intraperitoneal chemotherapy (HIPEC): Cytoreductive surgery showed a survival benefit in cases responded to preoperative imatinib. Debulking in the form of removal of the gross tumor followed by intraperitoneal chemotherapy [12] with cisplatin and doxorubicin or mitoxantrone; with median time to recurrence was increased from 8 to 21 months. GISTs may result in sarcomatosis that is chemotherapy-resistant, leaving patients with few options in the pre-TKI era. Disseminated GIST primary therapy is TKI therapy. TKI resistance in sarcomatosis in GIST is an important point to be considered and managed carefully before it develops. Cytoreduction should be considered in such cases. The median time to develop TKI resistance is approximately two years and only 20 per cent of patients will not progress.

Progression on TKIs is associated with poor outcomes even after complete cytoreduction [1-3,11]. Given a significant survival benefit of several years in favor of the TKI-responsive group. One surgical option that has been offered is cytoreductive surgery with heated intraperitoneal chemotherapy (CRS/HIPEC). This modality carries a prolonged recovery with morbidity rates approaching 40 per cent. As such, the role of surgery for metastatic GIST in the post-TKI era remains controversial. Recently, investigators have described the positive impact of re-section in selected patients with metastatic GIST. Most often these studies involve isolated peritoneal or liver metastases. Patients with peritoneal sarcomatosis represent a small subset of patients with metastasis and therefore are rarely analyzed as a unique cohort [1].

i. Hyperthermic intraperitoneal chemotherapy (HIPEC): The rationale for HIPEC originated from the potential benefits described for intraperitoneal (IP) therapy, the principles of HIPEC rely on using very high drug concentrations in direct contact with at risk lining surfaces and floating tumor cells, limited drug absorption, enhancing tumor drug exposure and limiting systemic toxicity. With mitomycin $\mathrm{C}$, we demonstrated peak IP concentrations that were about 50-fold higher than peak serum levels. Only HIPEC, or IP administration, is capable of achieving such high drug concentrations in vivo. [5-7,9]. The concept rely behind hyperthermiais to increase the intensity of therapy.

Action of agents for which heat was found to be synergistic in the laboratory, such as mitomycin C or cisplatin [1] variable degrees of bone marrow suppression have been demonstrated in a variety of series involving CS + HIPEC. Fortunately, however, only a small percentage of patients were seen to have grade IV marrow suppression in early clinical trials. HIPEC with mitomycin $\mathrm{C}$ has showed improved long-term survival in patients $[5,6]$.
There are two types of HIPEC have been described: open and closed $[5,6]$. In the open technique, plastic sheeting is attached to the edges of the abdominal incision and the operator move puts the belly to move contents around and uniformly distribute the chemotherapy solution. That fluid is perfused in and out with a heated pump perfusion circuit. But the operator will be in risk for exposure if there are any breaches in clothing.

In the closed technique, tubing is placed for inflow and outflow into the abdomen and pelvis, can be placed through the incision or percutaneously. Temperature probes are placed to monitor inflow and outflow temperatures. Temperatures can also be monitored from internal locations if desired. Typically, we have the chemotherapy solution flow into the pelvis or the upper abdomen and use the opposite location for outflow. Other configurations are possible.

The skin of the surgical incision is temporarily closed with a watertight suture. The size of the peritoneal surface can vary related to body surface area (about $60 \%$ of this area).Depend on the individual size, presence of ascites and the extent of resection the perfusion volume will be determined. As a general rule, usually around 3 liters for women and 4 liters for men. The drug is added to the circuit, once the heated perfusion has started, and it has been confirmed that there are no leaks and that the temperature is over $40^{\circ} \mathrm{C}$. However, the range of $37^{\circ} \mathrm{C}$ to $42^{\circ} \mathrm{C}$ has been safe in practice and is in the normothermiato-fever range. There is temporary rises in core temperatures during perfusion, typically in the range of $38.5^{\circ} \mathrm{C}$ to $39.5^{\circ} \mathrm{C}$, which rapidly revert to normal once the perfusion has terminated. The abdomen is gently shaken or kneaded during the perfusion in order to enhance uniform distribution. Variables from this point are the plateau or target inflow temperature, drug dosing (and in some cases re-dosing), and total time for perfusion.

Perfusion times range from 30 minutes to 2 hours, depending on the protocol. These formulae vary by institution, and some experts have called for standardization. However, all protocols have in common very high IP concentrations of the chemotherapeutic agent compared with serum levels. Different outcomes related to differences in drugs used; however, this has not been examined in clinical trials to date $[5,6]$. These procedures are applicable in same standards for GIST carcinomatosis.

\section{Discussion}

\section{Reported studies}

a. A prospective multi-institutional study from France known as EVOCAPE I was published in 2000. The aim of this study is to collect data from multiple institutions about patients who underwent CS but no HIPEC [5]. Patients received surgical treatment and systemic chemotherapy (including with oxaliplatin, available in France then) as it was the standard of care that time and place. The importance of this study that it showed that even in complete resection of disease R0, there were no long term five years survivals. The two year survival 
was only $10 \%$.The same year, Loggie et al. [5,7] published results of a prospective, National Institutes of Health-funded clinical treatment trial that showed an overall 5-year survival of 35\% in patients with GI peritoneal carcinomatosis treated with CS and HIPEC. Both studies showed that malignant ascites had a notable negative impact on survival.

b. Department of Surgery, National Cancer Institute, Milan, Italy (13). A prospective database of 37 patients who underwent CRS and close-abdomen HIPEC with cisplatin and doxorubicin or mitomycin-C was reviewed, From January 1996 to May 2006, PS (peritoneal sarcomatosis) originated from GIST (preimatinib era) in 8 patients, uterine leiomyosarcoma (ULS) in 11, retroperitoneal liposarcoma (RPLP) in 13, and other sarcoma in 5.For all patients, median overall survival was 26.2 months; 7 patients were alive at 46-130 months (ULS, $\mathrm{n}=4$; RPLP, $\mathrm{n}=2$; GIST, $\mathrm{n}=1$ ). Median survival was approximately 20 months for metastatic GISTs. At present, since imatinib has become the worldwide standard of treatment for advanced/ metastatic disease, median progression-free and overall survival has dramatically improved to approximately 2 and 5 years, respectively [13].

c. Surgical Oncology Service in the Departments of General Surgery, Winston-Salem, North Carolina, [10]. This is a study conducted retrospectively since 1992 to 2012, analysis of a prospective database of 1070 CRS/HIPEC procedures. The factors included in the study including, performance status, resection status, morbidity, mortality, perioperative use of targeted therapies, and overall survival. 18 CRS/HIPEC were performed for GIST induced sarcomatosis. Eligibility criteria were a histologic diagnosis of sarcomatosis, complete recovery from any previous treatment including systemic chemotherapy, radiation, surgery and no extra-abdominal disease.

Fifty per cent of these cases were performed before the introduction of TKIs. R0/1 resection was achieved in 72 per cent, whereas 63 per cent of patients were treated with neoadjuvant and/or adjuvant targeted therapy. Thirty-day morbidity and mortality were 33.3 and 5.6 per cent, respectively. Median overall survival after CRS/HIPEC was 3.33 years with 3 -year survival of 56 per cent. Median survival in those who did not receive targeted therapy was 1.04 versus 7.9 years for those treated with TKI and cytoreduction. Median postsurgical survival for those treated preoperatively with progression on TKI treatment was 1.35 years versus not reached in those on TKI therapy without progression. R0 for complete macroscopic and microscopic resection, R1 for complete macroscopic resection with residual microscopic disease, R2for residual macroscopic disease subdivided based on the size of residual disease (R2a 5 $\mathrm{mm}$ or less, R2b $2 \mathrm{~cm}$ or less, R2c greater than $2 \mathrm{~cm}$ ).

All data were collected prospectively and analyzed retrospectively. Patients were typically followed with physical examination and computed tomography imaging every 6 months.
Chemo perfusion was performed at $40^{\circ} \mathrm{C}$ with $40 \mathrm{mg}$ mitomycin C with or without 10 to $30 \mathrm{mg}$ mitoxantrone for 60 to 120 minutes. Results: 18 CRS/HIPEC procedures were performed in 16 patients for GIST-induced sarcomatosis. Mean age at the time of surgery was 49.7 years (range, 37 to 68 years). Sixtyone per cent of the patients had an ECOG functional status of 0 or 1 at the time of surgery. Mean albumin was 3.8 (range, 1.8 to 4.4), indicating that patients had adequate nutritional reserves at the time of surgery. A complete R0/R1 macroscopic CRS was obtained in 72 per cent (13 of 18). Six patients had a major Clavien III-IV complication, yielding an overall 30-day major morbidity rate of 33.3 per cent. There was one death within 30 days of surgery, yielding a 30-day mortality of 5.6 per cent. Median overall survival after CRS/HIPEC was 3.33 years (range, 0.64 to 11.9 years) for all patients regardless of TKI treatment. This study conducted before and after the introduction of TKIs.

\section{Importance of Patient Selection}

Unfortunately, there are few guidelines for the selection of patients for CS and HIPEC. For colorectal and appendiceal cancers, there is a general consensus bysurgical oncologists was published several years ago about patient selection. The ideal patient selection criteria includes, good performance status, no or minimal symptoms, and resectable diseasewith a "reasonable" operation. The final decision whether to proceed with HIPEC or no is made in the operating room [5,7]. Same will be applicable to our patients with carcinomatosis.

\section{Surveillance}

Since GISTs have unpredictable behavior, it is recommended to have long term follow up for all patients, independent of their nature of disease characteristics. As the recurrence of GISTs mostly within the first 3-5 years, intense follow up is required during this period. Clinic visits with physical examinationand abdomino pelvic CT scan every 3-6 months is the recommended surveillance protocol [1].

\section{Conclusion}

The role of cytoreductive surgery and hyperthermic intraperitoneal chemotherapy (CRS/HIPEC) in the management of patients with gastrointestinal stromal tumor (GIST) induced sarcomatosis that is refractory to tyrosine kinase inhibitors (TKI) is still not well defined. It may improve loco-regional disease control, but its role in improving survival in these patients remains uncertain, and should only be considered in the context of a prospective randomized clinical trial $[5,8]$.

\section{References}

1. Ashwin Rammohan, Jeswanth Sathyanesan, Kamalakannan Rajendran, Anbalagan Pitchaimuthu, Senthil-Kumar Perumal, et al. (2013) A gist of gastrointestinal stromal tumors: A review, World J Gastrointest Oncol 5(6): 102-112.

2. Joensuu H (2006) Gastrointestinal stromal tumor (GIST). Ann Oncol 10: $\mathrm{x} 280-\mathrm{x} 286$. 
3. Pidhorecky I, Cheney RT, Kraybill WG, Gibbs JF (2000) Gastrointestinal stromal tumors: current diagnosis, biologic behavior, and management. Ann Surg Oncol 7(9): 705-712.

4. Walker P, Dvorak AM (1986) Gastrointestinal autonomic nerve (GAN) tumor. Ultrastructural evidence for a newly recognized entity. Arch Pathol Lab Med 110(4): 309-316.

5. Brian W Loggie, Peter Thomas (2015) Gastrointestinal Cancers with Peritoneal Carcinomatosis: Surgery and Hyperthermic Intraperitoneal Chemotherapy. Oncology 29(7): 515-521.

6. Loggie BW, Sterchi JM, Rogers AT, et al. (1995) Intraperitoneal hyperthermic chemotherapy for advanced gastrointestinal and ovarian cancers. Reg Cancer Treat 7(2): 78-81.

7. Chu DZ, Lang NP, Thompson C, Osteen PK, Westbrook KC (1989) Peritoneal carcinomatosis in nongynecologic malignancy. A prospective study of prognostic factors. Cancer 63(2): 364-367.

8. Joel M Baumgartner, Steven A Ahrendt, James F. Pingpank, Matthew P Holtzman, Lekshmi Ramalingam, et al. (2013) Aggressive Locoregional Management of Recurrent Peritoneal Sarcomatosis. Journal of Surgical Oncology 107(4): 329-334.

This work is licensed under Creative Commons Attribution 4.0 Licens DOI: 10.19080/OAJS.2017.05.555662
9. Autumn J McRee, Bert H O'Neil (2015) The Role of HIPEC in Gastrointestinal Malignancies: Controversies and Conclusions, Published on Cancer Network.

10. Michelle L Bryan, Nora C Fitzgerald, Edward A Levine, Perry Shen, John H. Stewart, et al. Cytoreductive Surgery with Hyperthermic Intraperitoneal Chemotherapy in Sarcomatosis from Gastrointestinal Stromal Tumor, Am Surg 80(9): 890-895.

11. Andtbacka RH, Ng CS, Scaife CL, Hunt KK, Pisters PW, et al. (2007) Surgical resection of gastrointestinal stromal tumors after treatment with imatinib. Ann Surg Oncol 14(1): 14-24.

12. Sugarbaker PH (1995) Peritonectomy procedures. Ann Surg. 221(1) 29-42.

13. Dario Baratti, Elisabetta Pennacchioli, Shigeki Kusamura, Marco Fiore, Maria Rosaria Balestra, et al. Peritoneal Sarcomatosis: Is There a Subset of Patients Who MayBenefit from Cytoreductive Surgery and HyperthermicIntraperitoneal Chemotherapy? Ann Surg Oncol 17(12): 3220-3228.

14. Miettinen M, Lasota J (2003) Gastrointestinal stromal tumors (GISTs): definition, occurrence, pathology, differential diagnosis and molecular genetics. Pol J Pathol 54: 3-24.

\section{Your next submission with Juniper Publishers will reach you the below assets}

- Quality Editorial service

- Swift Peer Review

- Reprints availability

- E-prints Service

- Manuscript Podcast for convenient understanding

- Global attainment for your research

- Manuscript accessibility in different formats

( Pdf, E-pub, Full Text, Audio)

- Unceasing customer service

Track the below URL for one-step submission https://juniperpublishers.com/online-submission.php 